\title{
HOLOGRAPHIC RECORDING MEDIA BASED ON ELECTRONS DONOR OLIGOMERS
}

\author{
Nicolay Davidenko ${ }^{a^{*}}$, Irina Davidenko ${ }^{a}$, Nicolay Chuprina ${ }^{a}$, Yuriy Getmanchuk ${ }^{a}$, \\ Leonid Kostenko ${ }^{b}$, Elena Mokrinskaya a, Valeriy Pavlova, \\ Sergey Studzinsky ${ }^{a}$, Larisa Tonkopieva ${ }^{a}$ \\ ${ }^{a}$ Kiev Taras Shevchenko National University, 64, Volodymyrs'ka str., Kiev 01601, Ukraine \\ ${ }^{b}$ L.M. Litvinenko Institute of Physics Organic Chemistry and Coal Fuel Chemistry NAS of Ukraine \\ 70, R. Luksemburg str., Donetsk 83114, Ukraine \\ *email:ndav@univ.kiev.ua
}

\begin{abstract}
Information properties of the recording media for photothermoplastic technique with the films of oligomer composites based on carbazole containing cooligomers of linear and radial structures with branching center on silicon atoms are investigated. It was ascertained that the media based on the radial oligomers possess higher holographic sensitivity due to higher plasticity and ability to accumulate volume electric charge during the exposure. Examples of practical application of the investigated recording media are presented.
\end{abstract}

Keywords: carbazole, oligomer, photothermoplastic, holographic recording.

\section{Introduction}

Development of the new materials possessing photoconductivity in the visible and near IR parts of spectrum is very actual question for their practical applications in the equipment for recording, storage and processing of optical information. Along with inorganic semiconductors it seems to be attractive to use organic oligomers and based on them compositions (OC) [1-7]. These OC were tested in the holographic recording media (HRM) for the photothermoplastic technique (PTT) of information recording [8]. HRM for PTT holographic recording have to possess following characteristics: low electric conductivity; high absorption coefficient close to the irradiation maximum of used light sources; high quantum yield of photogeneration of charge carriers; high mobility of non-equilibrium charge carriers. Basically, photoconductivity of the OC films is provided by presence of the molecules of three types: donors, acceptors and centers of photogeneration of charge carriers [8,9]. Absorption of light quantum occurs in the photogeneration center resulting in its transition from the ground state into excited one. The dye molecules, the molecules of organic compounds with intramolecular charge transfer, the intermolecular complexes with charge transfer $(C C T)[9,10]$ can be considered as such centers. The excited state of the photogeneration center is characterized by empty limit orbitals, notably by presence of one electron on the high occupied molecular orbital (HOMO) and one electron on low unoccupied molecular orbital (LUMO). Photogeneration of charge carriers happens as the result of intermolecular electron transitions. Electron can transit from LUMO of the excited photogeneration center into LUMO of the molecule with electron acceptor properties. Electron from valent orbital of the molecule with electron donor properties can transit to empty HOMO of the photogeneration center. If the ionization potential of the electron donor molecules is $I_{\mathrm{gd}}$ (HOMO energy is proportional to this one) and affinity energy of the acceptor molecules is $A_{\text {ea }}$ [11] (LUMO energy is proportional to this one), then the molecules of photogeneration centers are chosen to satisfy the conditions $\left|I_{\mathrm{gc}}\right|>\left|I_{\mathrm{gd}}\right|,\left|A_{\mathrm{ec}}\right|<\left|A_{\mathrm{ea}}\right|$, where $I_{\mathrm{gc}}$ and $A_{\mathrm{ec}}$ are respectively ionization potential and affinity energy of the molecule of photogeneration centre. These conditions determine formation of electron - hole pair (EHP) in the molecule of photogeneration centre after absorption of light quantum and intermolecular electronic transitions. EHP can consist of either donor cation-radical and anion-radical of the photogeneration centre, or cation-radical of the phorogeneration centre and acceptor anion-radical, or donor cationradical and acceptor anion-radical. EHP dissociation involves appearance of free charge carriers.

The most important requirement of appearance of the photoconductivity effect in the OC films is existence of the charge carriers transport in the OC film volume [9]. The transport is provided by increase of the concentration of the donor and/or acceptor fragments in the OC films. The concentration growth enhances overlap of the wave functions of neighboring fragments and increases probability of intermolecular electron transitions. Oligomers with electron donor substitutes are used for production of the OC with hole conductivity type. $\pi$-conjugated closed systems (condensed aromatic cycles) can be employed as such substitutes. These systems are able to return electron but does not able to capture electron due to positive LUMO energy.

Aspects of influence of the oligomers structure on above notified properties are not clearly understood till now. Hetero-chain electron donor oligomers were synthesized and used before for production of photosensitive information media. That allows to develop HRM with optimal rheological properties. The oligomers do not possess high-elastic state. This fact allows to transit from solid vitreous state of the film into liquid state with low viscosity and backward very quickly. Time of development decreases until microseconds, the film functions in real time scale. The film has 
to possess enough elasticity for its using on flexible substrate. Such forced elasticity below the temperature of glass transition can be reached only due to conformational transitions in separate oligomer links consisting of, as a rule, donor multinuclear conjugated substitutes.

Not only structure of the macromolecule chain has significant influence on rheological properties of the polymers and oligomers, but also its shape, particularly branching. One can conclude from the results of our investigation that sensitivity and diffraction efficiency of the HRM considerably increases for the radial oligomers as compare to linear ones. Recently we have made [12] new oligomer: radial tetrasubstituted silan (RTS). Films of these oligomers possess improved rheological properties.

The present work is aimed at fulfilling of comparative analysis of information properties of HRM based on the oligomers with linear and radial molecular structures.

\section{Experiments}

Cooligomer of glycidylcarbazole with butylglycidyl ether (GCBE) of linear structure and radial oligomer RTS were used as the OC base:

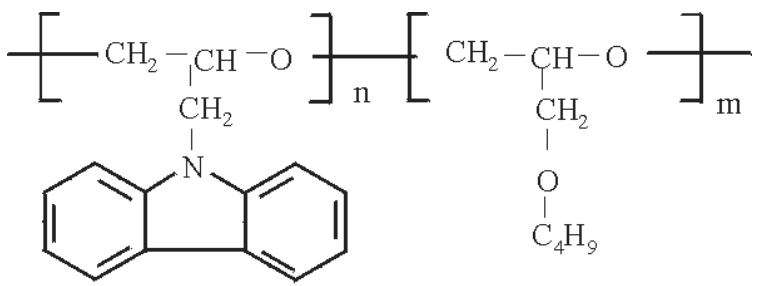

GCBE<smiles></smiles>

The synthesis of RTS was carried out according to the following scheme:

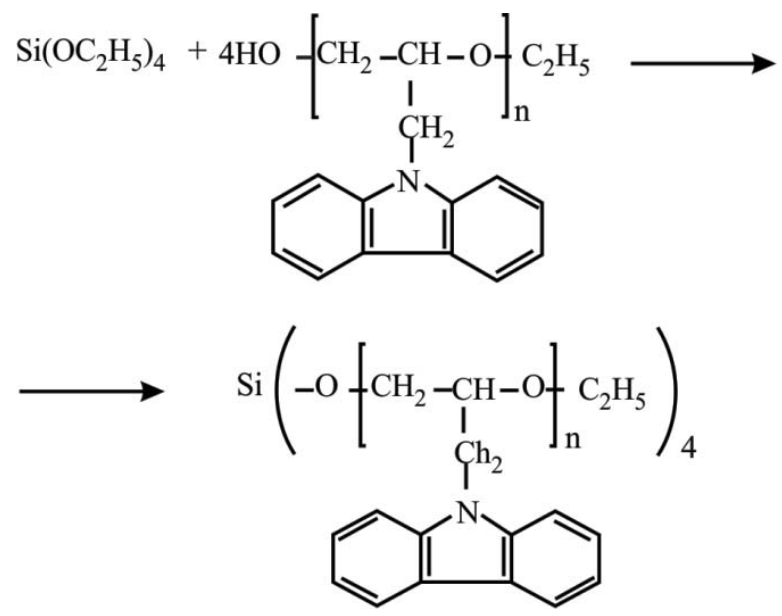

The $0.5 \mathrm{~g}(0.2 \mathrm{M})$ of tetraethoxysilane, $10 \mathrm{~g}(1 \mathrm{M})$ of oligoglycidylcarbazole, and $0.05 \mathrm{~g}$ of sodium was added to the four-neck $0.5 \mathrm{~L}$ reactor with a stirrer, the reflux condenser, and the thermometer. The starting compounds were dissolved in $5 \mathrm{~mL}$ of o-xylene and were heated (on boiling) and stirred for two hours. The residual xylene was distilled off, and then reaction mixture was separated from the sodium and xylene by washing with ethanol. The product was purified by recrystallisation from the mixture of benzene with petroleum ether or dry acetone. The main product is a white powder (yield $90 \%$, softening point $T_{\mathrm{m}}=95-105^{\circ} \mathrm{C}$ ). 
Acceptor undecyl ether 2,7- dinitro -9-dicyanmethylenefluorene-4-carboxylic acids (UE-DDFC) was used as the sensitizer of HRM photosensitivity:<smiles>CCCCCCOC(=O)c1cc([N+](=O)[O-])cc2c1-c1ccc([N+](=O)[O-])cc1C2=C(C#N)C#N</smiles>

Intermolecular complexes with charge transfer (CCT) arise while contacting acceptor UE-DDFC molecules with carbazole fragments of the oligomers (in liquid solutions and in solid films). Appearance of new wide absorption band is evidence of CCT arising. This band is absent in electronic spectra of OC individual components [9, 13]. The normalized spectra of optical density $(D)$ of the OC films based on GCBE, RTS and RTG with the same UE-DDFC are practically identical within visible range of spectrum (Figure 1). This is evidence of formation of identical CCT in these films.

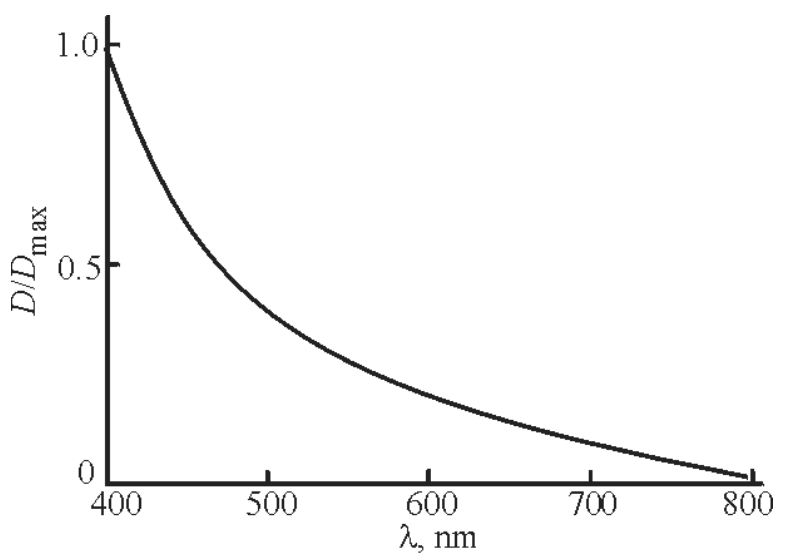

Figure 1. Normalized absorption spectrum of the films GCBE, RTS containing 3 mas.\% UE-DDFC.

For holographic recording by PTP method HRM were prepared similarly to $[4,10]$ as thin OC film deposited onto transparent conducting layer $\mathrm{SnO}_{2}: \mathrm{In}_{2} \mathrm{O}_{3}$ (ITO) with resistance $20 \mathrm{Ohm} /$ square. Ratio between the components in OC was following: oligomer - 97 mas.\%, UE-DDFC - 3 mas.\%. The thickness $(L)$ of the OC films was $1.1-1.2 \mu \mathrm{m}$ which is optimal for HRM.

Measurements of photothermoplastic characteristics of OC were done using known technique [14] of registration of the hologram of flat wave front. Spatial frequency was $500 \mathrm{~mm}^{-1}$, light wavelength of the used semiconductor laser was $650 \mathrm{~nm}$, ratio between light intensities in the reference and object beams was 1:1. Diffraction efficiency ( $\eta$ ) of the reconstructed image of the hologram of flat wave front in -1 diffraction order was measured continuously during development process. Photodetector was used for these purposes, signal from which was registered by memory oscilloscope Tektronix TDS1001B. The process of the hologram development was not ceased when maximal $\eta$ value was reached, heating of $\mathrm{OC}$ was continued during the duration of current pulse until full erasing of the hologram. Dependency of the maximal $\eta$ value on the exposure $I \cdot t$ was measured, where $I$ is the light intensity, $t$ is the time of the HRM exposure. Experiments on our small-sized holographic device [15] were carried out to test the possibilities of employing of the produced HRM in the technique of holographic interferometry.

\section{Results and discussion}

It was observed that the value $\eta$ increases at 1.5 times respectively in transition from HRM with GCBE to HRM with RTS (Figure 2). Maximal value $\eta$ was reached for short durations of the pulse of development of latent image for HRM with OC based on RTS as compare to HRM based on GCBE. This fact indicates that evolution of geometrical surface relief of the OC films with RTS during the hologram development occurs at lower temperatures as compared to GCBE. Low noise level of the reconstructed holographic images is evidence of good rheological properties of OC. 
However, growth of the holographic sensitivity at transition from HRM with OC based on GCBE to RTS can be attributed to once more reason. It was observed in experiments that investigated HRMs possess "memory" effect for preliminary illumination with light before charging of the surface of OC film in crown discharge. Investigations of the $\eta$ dependency on time $\left(t_{1}\right)$ of exposure before start of charging cycle and development of the latent holographic image, as well as $\eta$ dependency after long-time exposure of the HRM on time $\left(t_{2}\right)$ of delay before start of the charging cycle and development of the latent image were carried out. In these experiments only reference laser beam was used for measurements, the object beam was cut off. Before each next measurement charging of the surface of OC film was done in darkness and few long-time current pulses propagated for heating of the OC film. These operations allow being convinced in absence of the "memory" effect.

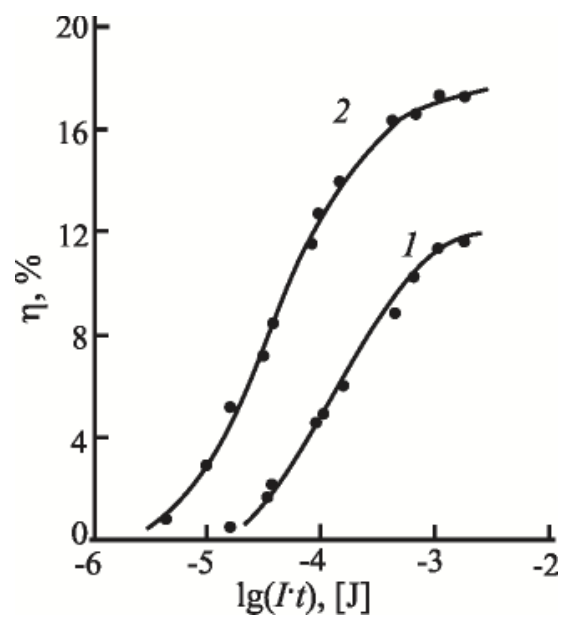

Figure 2. Dependencies of the maximal $\eta$ value on light energy absorbed in HRM during holographic recording for HRM based on GCBE (1) and RTS (2).

Additional investigations were fulfilled for ascertainment of the reason of the "memory" effect. The samples of sandwich structure glass substrate / ITO / OC film / Ag were prepared for these investigations. The concentration (Q) of non-equilibrium electric charge was measured in the samples. It appears in the volume of the OC film after its illumination with light without application of external electric voltage. Method of $Q$ determination and technique of measurement of $Q$ dependencies on time $\left(t_{1}\right)$ of illumination and time $\left(t_{2}\right)$ after illumination was ceased are illustrated by the diagrams in Figure 3.

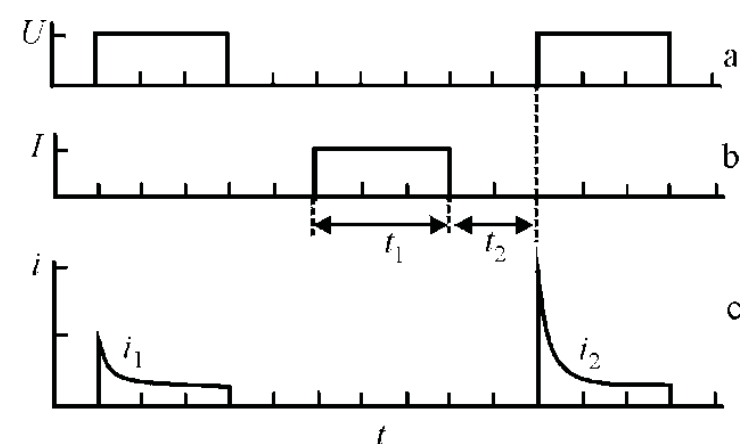

Figure 3. Diagrams demonstrating sequence of electric voltage application (a), illumination with light (b) and measurement of the current in the samples ITO / OC / Ag (c).

At first, electric voltage $(U)$ was applied to the sample in darkness and current $\left(i_{1}\right)$ kinetics was registered using memory oscilloscope. Next, electric contacts were disconnected and the sample was illuminated with light during the time $t_{1}$, light was switched off, after the time interval $t_{2}$ the electric contacts were connected, electric voltage $U$ was applied to the sample and current $\left(i_{2}\right)$ kinetics was registered. From these measurements $Q$ accumulation and relaxation were determined using relation $Q=\int\left(i_{2}-i_{1}\right) \mathrm{d} t / e S L$, where $e$ is the charge of electron, $S$ is the square of the sample of sandwich structure. The samples were illuminated from the side of ITO layer. $U$ value was measured within the range $20-300 \mathrm{~V}$. The measurements were done at room temperature. Dependencies of $\eta$ on durations $t_{1}$ and $t_{2}$ measured in the HRM samples with OC based on RTS and RTG are shown in Figure 4. 
Saturation of the "memory" effect (reaching of the stationary value in the dependency $\eta\left(t_{1}\right)$ ) occurs quicker (curve 1 in Figure 4) than its relaxation (dependency $\eta\left(t_{2}\right)$ ) (curve 2 in Figure 4). Besides, kinetic curves of the dependencies $\eta\left(t_{1}\right)$ and $\eta\left(t_{2}\right)$ are similar to the dependencies $Q\left(t_{1}\right)$ and $Q\left(t_{2}\right)$ (Figure 4). The value $Q$ increases with $U$ growth and does not depend on the polarity of applied voltage. The last fact testifies that the "memory" effect is attributed to accumulation and relaxation of the volume electric charge in the OC films as the result of their illumination with light. In the HRM samples with OC based on GCBE the "memory" effect and $Q$ accumulation were not observed.
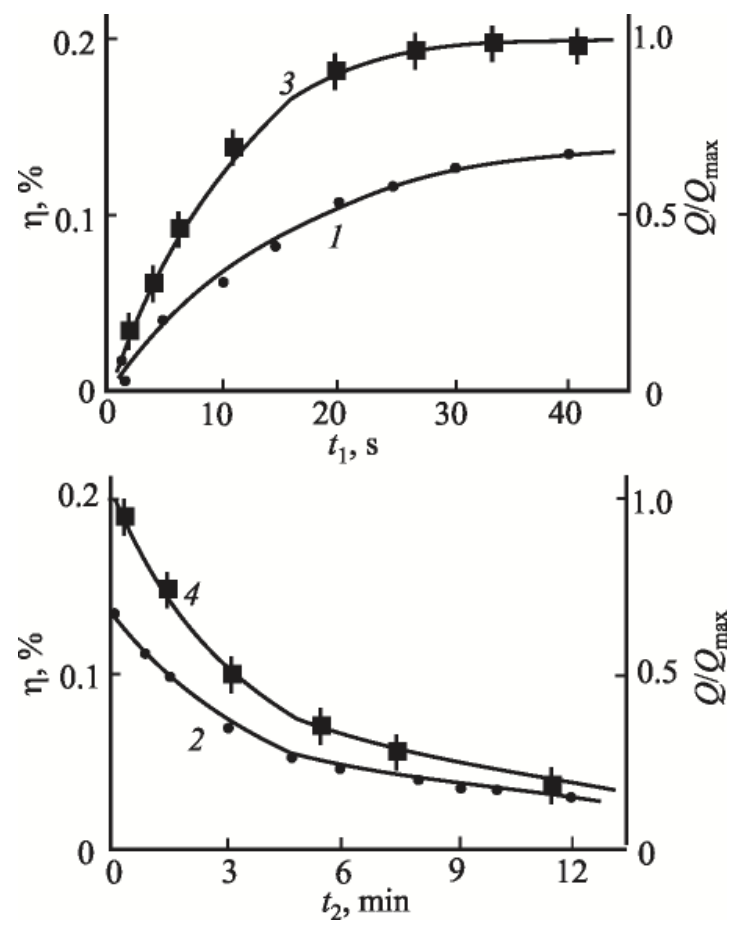

Figure 4. Dependencies $\eta\left(t_{1}\right)(1)$ and $\eta\left(t_{2}\right)(2)$ in HRM with OC based on RTS and dependencies $Q / Q_{\max }\left(t_{1}\right)(3)$ and $Q / Q_{\max }\left(t_{2}\right)(4)$ in the samples ITO / OC / Ag with OC based on RTS.

It is generally known that radial (starlike) polymers possess unusual low viscosity even having very high molecular mass. Radial polymers with hard chain always are more plastic as compare to their linear analogs even having significantly higher molecular mass. Similar properties are characteristic for oligomers also. The film of linear cooligomer starts to deform slowly within the wide temperature range $35-80^{\circ} \mathrm{C}$ and then flows quickly. The film of radial oligomer turns into liquid state quickly at temperature $\geq 60{ }^{\circ} \mathrm{C}$. These peculiarities involve increase of the sensitivity and diffraction efficiency of the material when radial oligomer is used. Small growth of the sensitivity for exposure time $\sim 1-2 \mathrm{~s}$ also can involve accumulation of the volume electric charge in the OC volume. However, under long-time illumination of the samples non-equilibrium electric charge appears in the OC films based on the radial oligomers. It relaxes during long time after light switching off. This volume charge can be leveled as a result of application of external electric field or by temperature increase.

Appearance of the volume electric charge in the OC films after their illumination with light can be attributed at least to two reasons. Firstly, if $\left|I_{\mathrm{gc}}\right|>\left|I_{\mathrm{gd}}\right|$ then after photoexcitation of the photogeneration centre and generation of hole on carbazolil oligomer fragment energetic barrier exists for opposite transition of non-equilibrium electron from the molecule of photogeneration centre to cation-radical of the carbazolil fragment. It is proportional to the difference of energies $\left|I_{\mathrm{gc}}\right|-\left|I_{\mathrm{gd}}\right|$. Such effect was observed before [16] when compounds with intramolecular charge transfer (CICT) were used as the photogeneration centers. $\left|I_{\mathrm{gc}}\right|>\left|I_{\mathrm{gd}}\right|$ for CICT. Not excited CICT molecules do not interact with the carbazolyl fragments of oligomers, absorption spectra of $\mathrm{OC}$ based on different oligomers practically coincide and are similar to absorption spectra of CICT liquid solutions. However, in our case CCTs consisting of carbazolyl fragments serve as the photogeneration centers. These fragments are similar to ones through which transport of non-equilibrium holes in external electric field occurs. As it was shown before, the barrier for holes recombination during EHP annihilation in such system is small and life time of geminate EHP is $\sim 10^{-7} \mathrm{~s}$.

The second reason can be attributed to energetic traps which are able to capture the photogenerated charges and hold them during long time. Physical dimers (excimers) of the carbazolyl fragments of sandwich structure can be considered as such traps. For them $\left|I_{\mathrm{gd}}\right|$ is $\sim 0.3 \mathrm{eV}$ less than in the carbazolyl fragments which are not included in the 
dimers [9]. In carbazolyl containing polymers and oligomers the dimers are formed by end carbazolyl groups due to more favorable steric location for interaction with neighboring fragments. In the molecules of radial oligomer RTS number of the end carbazolyl fragments is 2 times more as compare to linear GCBE. Therefore, probability of the dimers formation in the films of radial oligomers also is higher. This fact explains presence of the "memory" and formation of the long-living photoinduced volume electric charge in OC based on RTS and its absence (or much less value) in OC based on GCBE.

\section{Conclusions}

Fulfilled investigations testify that optimal properties of OC for GRM depend not only on the ratio between ionization potential of the donor and affinity of acceptor to electron but also on the shape of their macromolecules. Plastic and rheological properties of the films become better when radial oligomers are used instead linear ones. Increase of the number of end carbazolyl fragments in oligomer macromolecules provokes effect of holographic "memory". This effect is explained by accumulation of photoinduced volume charge in energetic traps formed by dimers of the end carbazolyl groups. This effect can be used for development of new information media, in particular for holographic recording with few exposures allowing to increase recording density. Besides, possibility of formation of long-lived photoinduced volume charge in the OC films is of interest for development of new materials for optoelectronics and molecular electronics.

\section{References}

1. Huang, C.; Wang, N.; Li, Y.; Li, C.; Li, J.; Liu, H.; Zhu, D. A new class of conjugated polymers having porphyrin, poly(p-phenylenevinylene), and fullerene units for efficient electron transfer. Macromolecules, 2004, 39, pp. 5319-5325.

2. Lanzi, M.; Paganin, L.; Caretti, D. New photoactive oligo- and poly-alkylthiophenes. Polymer, 2008, 49, pp. $4942-4948$.

3. Davidenko, N.A.; Ishchenko, A.A.; Kulinich, A.V.; Studzinsky, S.L. Effect of concentration of anionic polymethine dye in poly-N-epoxypropylcarbazole polymer film composite on the spectral-luminescent properties and photoconductivity. Spectrochimica Acta Part A: Molecular and Biomolecular Spectroscopy, 2012, 98, pp. 271-274.

4. Davidenko, N.A.; Dekhtyarenko, S.V.; Getmanchuk, Yu.P.; Ishchenko, A.A.; Kozinetz, A.V.; Kostenko, L.I.; Mokrinskaya, E.V.; Studzinskii, S.L.; Skryshevskii, V.A.; Skulskii, N.A.; Tretyak, O.V.; Chuprina, N.G. Photosemiconducting properties of holographic media based on ferrocenyl-containing cooligomers of glycidyl carbazole with these oligomers sensitized organic dye. Semiconductors, 2009, 43, pp. 1473-1478.

5. Davidenko, N.A.; Ishchenko, A.A.; Kozinets, A.V.; Kostenko, L.I.; Kurdyukova, I.V.; Mokrinskaya, E.V.; Studzinskii, S.L.; Chuprina, N.G. Sensitization of photoconductivity in ferrocene-containing oligomer by squarylium and merocyanine dyes. Journal of Applied Spectroscopy, 2011, 78, pp. 129-133.

6. Davidenko, N.A.; Dehtarenko, S.V.; Getmanchuk, Yu.P.; Ishchenko, A.A.; Kozinetz, A.V.; Kostenko, L.I.; Mokrinskaya, E.V.; Studzinskii, S.L.; Skryshevskii, V.A.; Tretyak, O.V.; Chuprina, N.G.; Sensitization of Photosemiconducting Properties of Holographic Recording Media Based on Glycidylcarbazole Cooligomers by Organic Dyes. Molecular Crystals and Liquid Crystals, 2011, 535, pp. 148-155.

7. Hariharan, P. Basics of Holography; Cambridge University Press: Cambridge, 2002, 411 p.

8. Davidenko, N.; Davidenko, I.; Ishchenko, A.; Kulinich, A.; Pavlov, V.; Studzinskii, S.; Chuprina, N. Reversible holographic recording media based on polymeric composites and their use in energy-saving technologies. Applied Optics, 2012, 51, pp. C48-C54.

9. Pope, M.; Swenberg, C. Electronic Processes in Organic Crystals and Polymers, 2nd ed; Oxford University: Oxford, 1999, $278 \mathrm{p}$.

10. Davidenko, N.; Ishchenko, A.; Kostenko, L.I.; Kuvshinskii, N.; Kulinich, A.; Melenevskii, D.; Mysyk, D.; Mysyk, R.; Pavlov, V.; Chuprina, N. Holographic Recording Media Based on Systems with Intramolecular and Intermolecular Charge Transfer. High Energy Chemistry, 2005, 39, pp. 254-262.

11. Danovich, D.; Apeloig, Y.; Shaik, S. A Reliable and Inexpensive Method for Calculating Ionization Potentials and Electron Affinities of Radicals and Molecules. Journal of Chemical Society, Perkin Trans., 1993, 2, pp. 321-330.

12. Getmanchuk, Yu.P.; Davidenko, N.A.; Kunitskaya, L.R.; Mokrinskaya, E.V. Information media based on electron donor oligomers. Polymer Science, Ser. B, 2013, 55, pp. 88-94.

13. Schaffert, R.M. Electrophotography; Wiley: New York, 1981, 318 p.

14. Rosen, J. ed. Holography, Research and Technologies; InTech: Rijeka (Croatia), 2011, 477 p.

15. Holographic Interferometer. http://www.youtube.com/watch?v=EZynG_qkGvM.

16. Davidenko, N.; Ishchenko, A.; Kuvshinskii, N. Photonics of Molecular Semiconductor Composites Based on Organic Dyes; Naukova Dumka: Kiev, 2005, 296 p. (in Russian). 